\title{
Exploring Early Stage Cost-Estimation Methods Using Off-the-Shelf Tools: A Preliminary Study
}

\author{
Haifeng Zhu, Narek Shougarian, Greg Ojard, Kaushik Sinha, \\ Oliver de Weck and Eileen Arnold
}

\begin{abstract}
Cost analysis is challenging for multiple reasons, one of which is the lack of historical data due to proprietary issues, or significant work required to make it useful for a particular application and domain of interest. In addition, to support system engineering methods such as Design Space Exploration, both componentand engine-level costs must be supported. This paper presents the results of a preliminary study on a tool that can be used to estimate the development cost for a set of airplane-engine architecture models using publicly available off-the-shelf tools. Our tool focuses on supporting complex system engineering tool chains and methods that require strong interoperability with different tools in a networked environment. The tool, through its architecture, allows the inclusion of supports for early stage cost analysis without directly using historical data, and both system- and component-level cost generations. We describe our approach, tools, estimation process and possible use cases.
\end{abstract}

Keywords Cost-estimation • Early stage

\footnotetext{
This research was partially developed with funding from the Defense Advanced Research Projects Agency (DARPA) and the Air Force Research Laboratory (AFRL). The views, opinions, and/or findings expressed are those of the author(s) and should not be interpreted as representing the official views or policies of the Department of Defense or the U.S. Government. Distribution Statement "A” (Approved for Public Release, Distribution Unlimited).
}

\section{H. Zhu (凶) · G. Ojard}

United Technologies Research Center, 411 Silver Lane, East Hartford, CT 06018, USA

e-mail: zhuhf@utrc.utc.com

N. Shougarian · K. Sinha $\cdot$ O. de Weck

Massachusetts Institute of Technology, 77 Massachusetts Ave. Building E38-532/33-410,

Cambridge, MA 02139, USA

E. Arnold

UTC Aerospace Systems, 4747 Harrison Ave., Rockford, IL 61108, USA

(C) Springer International Publishing AG 2017

G. Fanmuy et al. (eds.), Complex Systems Design \& Management,

DOI 10.1007/978-3-319-49103-5_28 